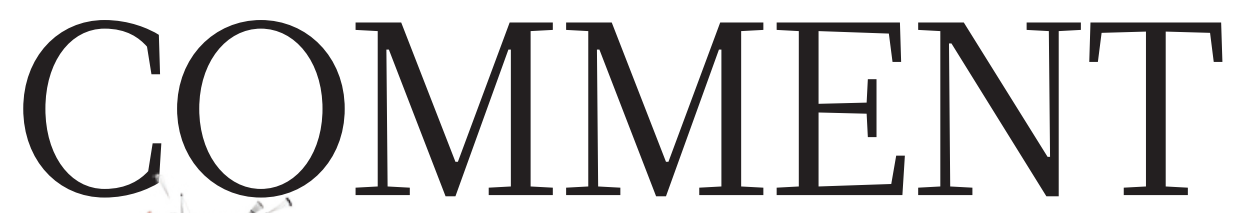

\title{
xion:
}

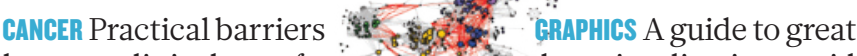
hamper clinical use of data visualization to aid genomic data $\mathbf{p . 2 9 0}$
HISTORY In praise of William

Smith, the first to map a

nation's geology p.294
FUNDING Romanian scientists feeling the pinch of EU nuclear-physics project $\mathbf{p . 2 9 5}$

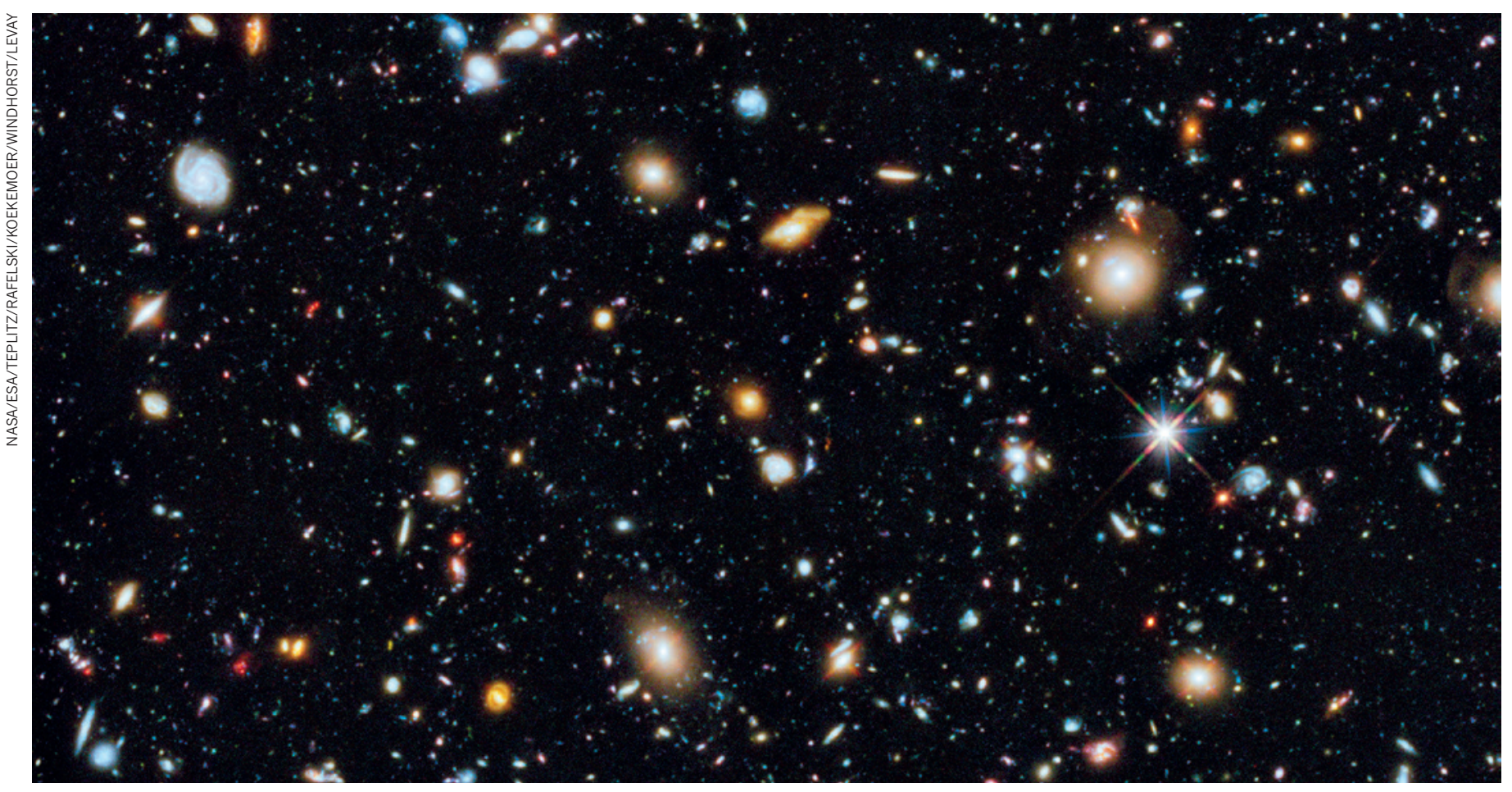

The Hubble Ultra Deep Field 2014 combines the full range of wavelengths available to the telescope, from ultraviolet to near-infrared.

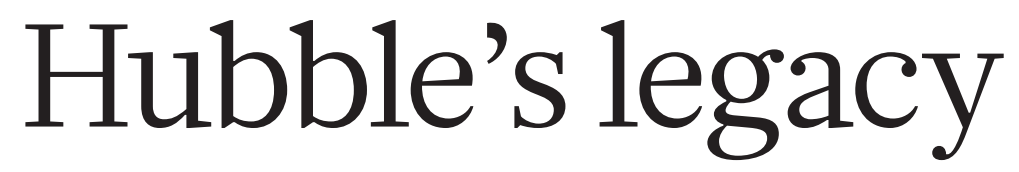

Twenty-five years after launch, the wild success of the space telescope argues for a new era of bold exploration in the face of tight budgets, says Mario Livio.

$(2$ n 24 April, it will be 25 years since the Hubble Space Telescope (HST) was launched from Cape Canaveral, Florida, into low-Earth orbit aboard the space shuttle Discovery.

As well as revolutionizing astrophysics, the first major optical observatory in space - built by NASA with contributions from the European Space Agency (ESA) - has brought the excitement of scientific discovery into millions of homes. Ask people to name a telescope and most will probably say "Hubble".

Circling the Earth every hour and a half, the observatory has completed more than 130,000 orbits and taken more than 1 million exposures of astronomical objects, from dust clouds to distant galaxies. More than 12,800 scientific articles have used HST results, and have been cited more than 550,000 times, making the telescope one of the most productive scientific instruments ever built.

What are the secrets of Hubble's success? Its longevity, pioneering of open data, superior archiving, attention to community needs, dedicated teams of space agencies, astronauts, scientists and engineers, and outstanding outreach infrastructure are all key. These have transformed what seemed initially to be a gigantic failure - flaws in the primary mirror were revealed within weeks - into a scientific triumph.

As Hubble enters its final productive decade, and successors such as the James Webb Space Telescope (JWST) inch towards the launch pad, it is a good time to reflect on its legacy and lessons (see 'Hubble's hits and beyond'). Hubble has taught us that to answer the most intriguing questions in astrophysics, we must think big and put scientific ambition ahead of budgetary concerns. In my view, the next priority should be the search for life beyond our Solar System. A powerful space telescope that can spot biological signatures in the atmospheres of Earth-like exoplanets would be a worthy successor.

\section{ALL IN THE DETAILS}

Hubble's greatness lies not so much in the singular discoveries that it has made as in confirming suggestive results from other observatories. As new details have become visible, astrophysicists have had to refine $>$ 


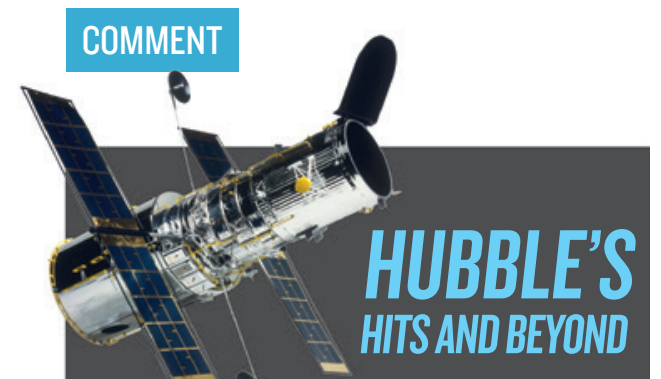

In-flight servicing has prolonged the space telescope's life, paving the way for future missions.

\section{0}

The Hubble Space Telescope is

launched on the space shuttle Discovery on 24 April. Distortions in the mirror are discovered on 25 June.

\section{3}

In the first servicing mission, astronauts fix the optics and install a new camera.

1996

The first Hubble Deep Field image is released, showing far-flung galaxies.

$1997,1999 \& 2002$

Servicing missions add a spectrograph and an infrared camera, fix worn gyroscopes that keep the telescope pointing correctly, and replace a camera and solar panels.

\section{4, 2007}

Power supplies fail on the spectrograph (2004) and on a camera (2007).

\section{8}

Hubble shows exoplanet Fomalhaut $\mathbf{b}$ and completes its hundred-thousandth orbit of Earth.

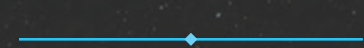

\section{9}

Astronauts carry out extensive repairs, and install a new camera and spectrograph.

\section{1}

Hubble makes its millionth observation (of an exoplanet) and the ten-thousandth scientific paper using its data is published (concerning supernovae).

\section{8}

The James Webb Space Telescope will open up infrared views of the Universe.

\section{$\sim 2024$}

WFIRST/AFTA will enable large surveys in the infrared from space.

\section{$\sim 2030$}

Proposed launch of a new major observatory to image and characterize exoplanets. their theories about the Universe.

The telescope's power stems from its high perch above most of Earth's atmosphere, at an altitude of about 560 kilometres. Unaffected by airglow (faint light emitted by atmospheric chemical processes) and turbulence, Hubble has a sharp eye (resolution) and can detect faint objects (sensitivity), even though its 2.4-metre-diameter mirror is small by today's standards (8-10-metre mirrors are now the norm). It can resolve objects 0.07 arcseconds apart - akin to reading the year on a dime from three kilometres away. That is ten times finer in visible light than any ground-based observatory can achieve.

The telescope sees wavelengths from ultraviolet to the near-infrared, including bands that are blocked by the atmosphere to astronomers on Earth. Its capabilities in the ultraviolet are about 100 times greater than those of its predecessors or of any current telescope.

The original plan for Hubble was for it to tackle three major problems: measure how fast the Universe is expanding, work out how galaxies evolve, and probe the structure of diffuse gas clouds lying between galaxies (the intergalactic medium). It has succeeded and provided unexpected sights along the way. Here is my selection of a few of Hubble's most important scientific achievements.

\section{GREATEST HITS}

One of the telescope's first jobs was to reduce the uncertainty in the cosmic expansion rate - the 'Hubble constant' - named, like the telescope, after its discoverer Edwin Hubble. Between 1994 and 2011, the uncertainty was reduced from a factor of 2 to a few per cent. Hubble thus helped to set the age of the Universe at 13.8 billion years. It did so by extending to more remote galaxies an established method of inferring distances from the cycles of changing brightness in a class of pulsating stars known as Cepheid variables.

The HST confirmed in 1998 that the cosmic expansion is accelerating, propelled by a mysterious form of 'dark energy'. This feat was achieved by monitoring supernovae - exploding stars - that are out of the reach of ground-based telescopes. Understanding the nature of dark energy is one of the most important challenges that physicists face.

The telescope also produced an 'executive summary' of star formation across cosmic time. In a series of roughly tenday observations between 1995 and 2014, it peered intently at small patches of sky, reaching deeper than any instrument has gone before. The resulting images are collectively known as the Hubble Deep Fields. Finding that many galaxies already existed 500 million years after the Big Bang, the HST challenged ideas about how the first stars formed, heated and re-ionized the Universe. Astronomers are still trying to fully understand why the rate at which new stars were born peaked about 10 billion years ago.

Using its high resolution to observe the motions of stars and gas in the centres of galaxies, the Hubble telescope proved that almost all galaxies have at their heart a supermassive black hole (with masses millions to billions of times that of the Sun). The mass of the black hole scales with that of the 'bulge' of stars surrounding it, showing that galaxies and black holes evolved together.

The HST also determined for the first time the chemical composition of the atmospheres of some giant extrasolar planets, revealing in 2001 the spectral signatures of elements such as sodium and in 2008 molecules such as water and methane. A larger telescope might one day be able to identify signatures of life processes - such as oxygen and chlorophyll - in the atmospheres of rocky planets beyond our Solar System.

\section{SECRETS OF SUCCESS}

Scientific prowess is not the sole reason for Hubble's success. Five servicing missions - in 1993, 1997, 1999, 2002 and 2009 - by spaceshuttle astronauts allowed the telescope to be reinvented. Astronauts have introduced corrective optics, replaced mechanical tape recorders with solid-state memory drives, upgraded the solar arrays and installed cameras and spectrographs. Without those repairs, Hubble would not be working today, or would be operating with 1970s technology.

Chance favours the prepared. Four more factors have multiplied the HST's productivity: making data rapidly and openly available; effective and accessible archiving; undertaking risky projects; and a robust funding and fellowship system.

Creative thinking was championed through reserving $10 \%$ of observing time for very large, time-critical or unconventional proposals at the director's discretion. The original Hubble Deep Field imaging, for instance, was advocated and led by Robert Williams, then director of Hubble's scientific operator, the Space Telescope Science Institute (STScI). Other observatories, including the Gemini Observatory in Hawaii and Chile and the Large Binocular Telescope in Arizona, have adopted the approach.

Researchers are given a year to analyse Hubble observations before the data are made public. Special data sets such as the Hubble Deep Fields were made openly accessible immediately. The HST was not the first space observatory to adopt this policy, but it inspired others to follow suit: the data from the Swift Gamma-Ray Burst Mission, launched in 2004, for example, are immediately available.

From the start, the archiving and dissemination of data were more rigorous and more highly automated (including calibrated data, for example) than at other observatories. For the past decade, more archive-based papers 
have been published each year than ones using proprietary data: in 2014, 302 papers relied on archival data alone; 283 used proprietary data. The European Southern Observatory adopted the HST archiving practices in 1993.

All allocated HST observations come with a NASA research grant, to ensure that the data are analysed and the results published quickly. Since 1990, more than 4,600 HST proposals have been accepted, and grants awarded totalling US $\$ 500$ million.

The project has also sponsored a new generation of top researchers. Since 1990, there have been 352 Hubble fellows - postdoctoral researchers who are funded to work independently for three years on Hubble-related science at US universities. Since 1993, about $500 \mathrm{PhD}$ theses have used Hubble data.

\section{THE PEOPLE'S TELESCOPE}

The HST has transformed the landscape of scientific outreach and education. An STScI Office of Public Outreach was funded almost from the start to offer press releases, online outreach and education to schools, science centres and planetariums. Embedding the office in the STScI - located on the campus of Johns Hopkins University in Baltimore, Maryland - ensured that professional astronomers were involved. An attractive and user-friendly website (hubblesite.org) attracts billions of hits a year.

Hubble educators pioneered the online dissemination of materials to schools, starting at a time when little was available. Today its materials reach more than 6 million students and 500,000 educators each year in the United States alone. Multimedia presentations on galaxies, exoplanets and black holes play in science centres worldwide.

Hubble images - dubbed by British art critic Jonathan Jones "the most flamboyantly beautiful artworks of our time" - have infiltrated general culture. A dedicated team ensures their visual quality. HST views have been included in art exhibits from Baltimore to Venice. They adorn book covers and music albums, such as Binaural by the rock

"For the first time in human history, an answer to the question 'Are we alone?' is within reach."

band Pearl Jam, have inspired contemporary classical music (such as The Hubble Cantata by composer Paola Prestini) and dance performances.

\section{NOW WHAT?}

The Hubble has shown that it is better to fund the right experiment fully than to compromise to fit a tight budget. Likewise, future major astronomical endeavours should: identify the most important question that needs to be answered; determine what it would

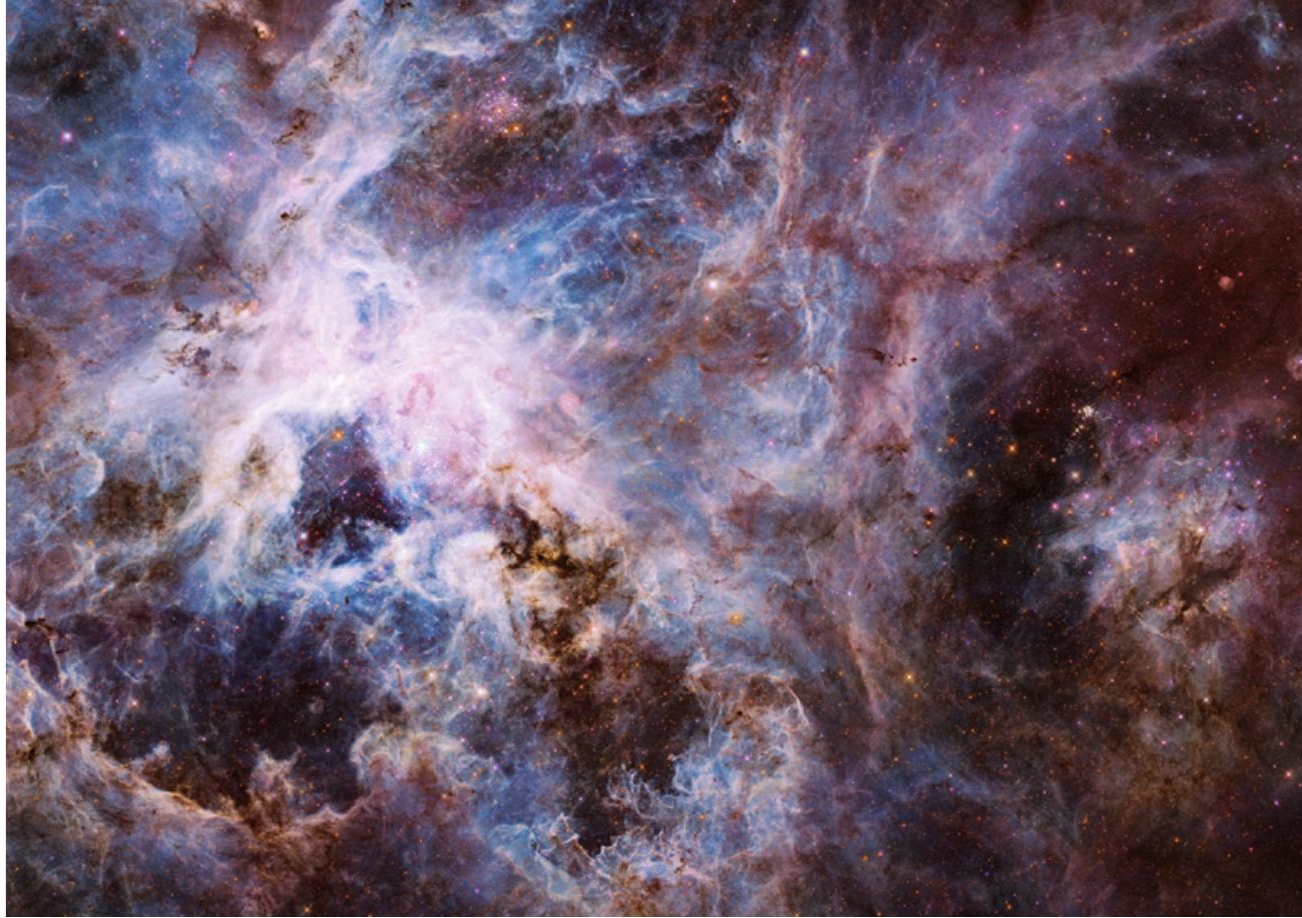

The Tarantula nebula, snapped by Hubble in visible, infrared and ultraviolet light.

take to answer the question and the technical feasibility of doing so; estimate the full cost of such a project; evaluate whether the goal is worth the investment; and act accordingly. That is, establish the necessary funding profile and keep it stable. Avoid cost overruns through careful planning and oversight.

The most intriguing question in astronomy is, in my view, whether life exists in our Galaxy beyond the Solar System. Thanks especially to the Kepler space telescope, we know that the Galaxy is teeming with hundreds of millions of Earth-sized planets in the 'habitable zones' of their host stars that allow for liquid water on a rocky surface.

The next steps are laid out. When it is launched in 2017, the Transiting Exoplanet Survey Satellite (TESS) should find a handful of nearby planets slightly heavier than Earth in the habitable zones of low-mass stars. The orbital periods of such planets are short and their stars faint, making them somewhat easier to detect. Then, the JWST, to be launched in 2018, and the Wide Field Infrared Survey Telescope-Astrophysics Focused Telescope Assets (WFIRST/AFTA), planned for around 2024, should look for water and other molecules in the atmospheres of a few of these planets.

A more powerful telescope will be needed to place meaningful statistical constraints on how common or rare life in the Galaxy is. One with a mirror at least 12 metres across and with a resolution 25 times that of Hubble's would be able to image a planet next to its star and detect spectrally the presence of oxygen and other biosignatures in its atmosphere. WFIRST/AFTA should be able to detect a planet 1 billion times fainter than its star; a brightness contrast of 10 billion will be required to image an Earth analogue next to a Sun-like host star. Clearly, such a telescope would offer a plethora of other discoveries as well.
A large sample of planets - around 50 would have to be tested. Calculations show, for example, that if no biosignatures are detected in more than about three dozen Earth analogues, the probability of remotely detectable extrasolar life in our Galactic neighbourhood is less than about $10 \%$.

A report on such a 'high-definition' telescope is expected to be published around June by the Association of Universities for Research in Astronomy. Several steps should be taken now. First, NASA, ESA and other potential international partners should convene a panel to examine such a project. Technology-development studies should be accelerated to make a launch around 2030 plausible. The search for life must be prioritized in the next US and international decadal surveys that guide national funding decisions about missions. The US astronomical community will recommence those discussions in 2016 for research priorities in the next decade.

In the meantime, I would also welcome substantially increased investment in the Search for Extraterrestrial Intelligence (SETI) project. Around \$100 million in extra funding, perhaps from private sources, would speed up the survey to a point at which about 10 million stars could be searched in a decade for radio or optical signals that are indicative of intelligent life. The chance of success may be low, but the pay-off could be huge.

For the first time in human history, an answer to the question 'Are we alone?' is within reach. The search for life should be high on the scientific agenda for the next 25 years. $\square$ SEE NEWS FEATURE P.282

Mario Livio is an astrophysicist at the Space Telescope Science Institute (STScI) in Baltimore, Maryland, USA.

e-mail:mlivio@stsci.edu 